\title{
Development and validation of an immune gene-set based prognostic signature for soft tissue sarcoma
}

Rui Shen ${ }^{1 \dagger}$, Bo Liu ${ }^{1 \dagger}$, Xuesen $\mathrm{Li}^{1 \dagger}$, Tengbo Yu${ }^{2}$, Kuishuai $\mathrm{Xu}^{2}$ and Jinfeng $\mathrm{Ma}^{1 *}$

\begin{abstract}
Background: Sarcomas is a group of heterogeneous malignant tumors originated from mesenchymal tissue and different types of sarcomas have disparate outcomes. The present study aims to identify the prognostic value of immune-related genes (IRGs) in sarcoma and establish a prognostic signature based on IRGs.

Methods: We collected the expression profile and clinical information of 255 soft tissue sarcoma samples from The Cancer Genome Atlas (TCGA) database and 2498 IRGs from the ImmPort database. The LASSO algorithm and Cox regression analysis were used to identify the best candidate genes and construct a signature. The prognostic ability of the signature was evaluated by ROC curves and Kaplan-Meier survival curves and validated in an independent cohort. Besides, a nomogram based on the IRGs and independent prognostic clinical variables was developed.

Results: A total of 19 IRGs were incorporated into the signature. In the training cohort, the AUC values of signature at 1-, 2-, and 3-years were 0.938, 0.937 and 0.935, respectively. The Kaplan-Meier survival curve indicated that highrisk patients were significantly worse prognosis $(P<0.001)$. In the validation cohort, the AUC values of signature at 1-, 2-, and 3-years were 0.730, 0.717 and 0.647, respectively. The Kaplan-Meier survival curve also showed significant distinct survival outcome between two risk groups. Furthermore, a nomogram based on the signature and four prognostic variables showed great accuracy in whole sarcoma patients and subgroup analyses. More importantly, the results of the TF regulatory network and immune infiltration analysis revealed the potential molecular mechanism of IRGs.
\end{abstract}

Conclusions: In general, we identified and validated an IRG-based signature, which can be used as an independent prognostic signature in evaluating the prognosis of sarcoma patients and provide potential novel immunotherapy targets.

Keywords: Soft tissue sarcoma, Immune-related gene, Nomogram, Immune infiltration

\section{Background}

Sarcoma is a rare group of heterogeneous malignant tumors originated from mesenchymal tissue, mostly occurring in the soft tissue and ending of the long bone [1]. Sarcoma can be classified into more than 80

\footnotetext{
*Correspondence: majinfengqddx@yeah.net

${ }^{\dagger}$ Rui Shen, Bo Liu and Xuesen Li contributed equally to this work.

'Department of Spinal Surgery, the Affiliated Hospital of Qingdao University, Qingdao 266000, China

Full list of author information is available at the end of the article
}

histological types according to the histological and molecular features [2]. Osteosarcoma, leiomyoma, lymphosarcoma, and synovial sarcoma are four common histological types [3]. Although sarcoma only accounts for $1 \%$ of malignancies, they account for $10 \%$ $12 \%$ of malignancies in children and adolescents $[2,4,5]$. The incidence in recent years is $2.49 \sim 5.87$ per person-year [6-9] and the 5-years survival rate after diagnosis was $56.4 \% \sim 61.6 \%$ [6-8]. However, $40 \% \sim 50 \%$ of sarcoma

(c) The Author(s). 2021 Open Access This article is licensed under a Creative Commons Attribution 4.0 International License, which permits use, sharing, adaptation, distribution and reproduction in any medium or format, as long as you give appropriate credit to the original author(s) and the source, provide a link to the Creative Commons licence, and indicate if changes were made. The images or other third party material in this article are included in the article's Creative Commons licence, unless indicated otherwise in a credit line to the material. If material is not included in the article's Creative Commons licence and your intended use is not permitted by statutory regulation or exceeds the permitted use, you will need to obtain permission directly from the copyright holder. To view a copy of this licence, visit http://creativecommons.org/licenses/by/4.0/ The Creative Commons Public Domain Dedication waiver (http://creativecommons.org/publicdomain/zero/1.0/) applies to the data made available in this article, unless otherwise stated in a credit line to the data. 
patients could develop metastasis $[3,10]$, which makes it difficult to choose an appropriate treatment, such as surgery, chemotherapy, and radiotherapy. Therefore, it's important to find effective makers for risk assessment for sarcoma patients.

Recently, a number of markers, including alternative splicing events, lncRNA, and miRNA, have been identified as prognostic biomarkers for sarcoma patients [1114]. Unfortunately, these potential biomarkers have been unable to be used in clinical practice, which may be caused by the weak prognostic ability and lacking validation. Lately, a large number of studies were performed to investigate the role of immune-related features in malignant tumors. Changes in the immune system have been shown to play an important role in tumorigenesis and development [15]. For this, immunotherapy is considered as a new and powerful therapy, especially for targeting programmed cell death 1(PD1) and Programmed Death Ligand-1(PDL$1)$ techniques. By acting on the immune checkpoints, it has been used to treat a variety of cancers, including sarcoma [16]. More importantly, previous studies indicated that immune-related genes (IRGs) can serve as an effective prognostic biomarkers in many tumors, such as urological cancer [17, 18], digestive cancer [19-22] and non-small cell lung cancer [23-26]. Nonetheless, the role of IRGs in sarcoma remains unknown but urgent.

Therefore, the aim of our study was to study the prognostic value of IRGs in sarcoma cohort based on the RNA-sequencing and clinical data from TCGA-SARC (https://cancergenome.nih.gov/) and the IRGs from ImmPort database (https://www.immport.org/shared/). In addition, the regulatory network between prognostic transcription factors (TFs) and prognostic IRGs was established. Finally, a nomogram based on the IRGs and prognostic clinical variables was developed and evaluated.

\section{Methods}

\section{Patient samples and IRGs}

RNA-sequencing (RNA-seq) expression profile and corresponding clinical information of soft tissue sarcoma patients were downloaded from TCGA database, and the clinical information of all patients are shown in Table 1. Meanwhile, the IRG set was obtained from the ImmPort database, which covered 2498 genes, including antigen processing and presentation, antimicrobials, B cell antigen receptor (BCR) signaling pathway, chemokines, chemokine receptors, etc. Then, 170 patients $(2 / 3)$ were randomly selected to form the training set and the remaining 85 patients $(1 / 3)$ were incorporated into the testing set.

\section{Identification of OS-related IRGs in sarcoma patients and enrichment analysis}

To identify the prognostic value of IRGs in sarcoma patients, we performed the univariate Cox proportional hazard model to identify the overall survival (OS)-related IRGs in the training set, and genes with a $p<0.05$ were considered as the OS-related genes. Meanwhile, to further understand the function of OS-related IRGs, Gene Ontology (GO), including molecular function (MF), biological process (BP), and cellular component (CC) and Kyoto Encyclopedia of Genes and Genomes (KEGG) database were used for enrichment analysis. The GO and KEGG analyses were performed by the $\mathrm{R}$ package clusterprofiler [27].

\section{Construction of a transcription factor regulatory network}

To determine the possible mechanisms behind the regulation of OS-related IRGs in soft tissue sarcoma patients, we analyzed the correlation between the expression profile data of OS-related TFs and OS-related IRGs. TF set was downloaded from the Cistrome Cancer (http:// cistrome.org/). Then, we used univariate Cox proportional hazard model to detect OS-related TFs. The correlation between OS-related TFs and OS-related IRGs were performed by the Pearson correlation analysis. Correlation with a $p<0.01$ and $r>0.5$ was considered to be statistical significance. To better illustrate regulatory relationships between the TFs and IRGs, TF-based regulatory network was generated by Cytoscape [28].

\section{Construction and evaluation of IRGs prognostic signature}

Based on the OS-related IRGs identified in the univariate Cox proportional hazard model, we performed the LASSO analysis to avoid overfitting [29]. Then, the significant genes in the LASSO regression were incorporated into the multivariate Cox analysis to detect the ultimate prognostic IRGs of the signature. A prognostic signature was established based on ultimate prognostic IRGs of the signature and the risk score was acquired according to the following formula:

$$
\text { Risk Score }=\sum_{i=0}^{n} \beta_{i} * \mathrm{G}_{i}
$$

Here, ' $G_{i}$ ' is the expression of the selected gene, and ' $\beta_{i}$ ' is the coefficient of the gene from the multivariate Cox proportional hazards analysis.

To identify the prognostic ability of the IRGs-based signature, time-dependent receiver operating characteristic (ROC) curves were generated, and the corresponding time-dependent area under the curve (AUC) was calculated simultaneously. In addition, 170 patients were stratified into the high- and low-risk group based on the 
Table 1 Clinical characteristics of sarcoma patients

\begin{tabular}{|c|c|c|c|c|}
\hline & Training set & Validation set & $t / X^{2}$ & $\mathbf{P}$ \\
\hline Age & $60.74 \pm 15.29$ & $60.46 \pm 13.50$ & 0.141 & 0.888 \\
\hline Sex & & & 0.198 & 0.657 \\
\hline Male & 79 & 37 & & \\
\hline Female & 91 & 48 & & \\
\hline Race & & & 1.074 & 0.783 \\
\hline Asian & 5 & 1 & & \\
\hline African American & 11 & 7 & & \\
\hline WHITE & 149 & 75 & & \\
\hline NA & 5 & 2 & & \\
\hline Histological type & & & 4.753 & 0.314 \\
\hline LMS & 65 & 37 & & \\
\hline DLP & 34 & 23 & & \\
\hline UPS & 35 & 14 & & \\
\hline MYX & 18 & 7 & & \\
\hline Other & 18 & 4 & & \\
\hline Metastasis & & & 1.282 & 0.527 \\
\hline No & 84 & 36 & & \\
\hline Yes & 34 & 21 & & \\
\hline NA & 52 & 28 & & \\
\hline Surgical margin resection status & & & 0.301 & 0.860 \\
\hline Ro & 104 & 49 & & \\
\hline R1-2 & 49 & 27 & & \\
\hline NA & 17 & 9 & & \\
\hline Radiotherapy & & & 0.672 & 0.715 \\
\hline No & 94 & 43 & & \\
\hline Yes & 46 & 27 & & \\
\hline NA & 30 & 15 & & \\
\hline Multifocal indicator & & & 0.699 & 0.705 \\
\hline No & 131 & 64 & & \\
\hline Yes & 24 & 15 & & \\
\hline NA & 15 & 6 & & \\
\hline
\end{tabular}

LMS Leiomyosarcoma, DLP Dedifferentiated liposarcoma, UPS Undifferentiated pleomorphic sarcoma, MYX Myxofibrosarcoma

median of risk score. Kaplan-Meier (K-M) survival curve and log-rank test was performed to show the difference of survival status between two groups [30].

\section{External validation of IRGs prognostic signature}

According to the prognostic signature developed in the training set, risk scores of 85 patients in the testing set were calculated. and all patients were classified into high- and low-risk groups. Similarly, K-M survival curves with log-rank test and ROC analysis were utilized to evaluate the accuracy of the prognostic signature in an independent validation cohort.

\section{Development of a nomogram based on IRGs and clinical variables}

In the present study, the data of age, sex, race, histological type of tumor, tumor site and patient metastatic status were obtained from TCGA portal. To investigate whether the risk model is independently associated with the prognosis of soft tissue sarcoma patients, univariate Cox analysis was performed to identify the prognostic variables, which were incorporated into the multivariate Cox analysis to determine the independent prognostic factors. Furthermore, based on the independent prognostic factors, a prognostic nomogram was established [31]. C-index, calibration curve, and decision curve 
analysis (DCA) were used to evaluate the performance of the nomogram [32].

\section{Identification of immune infiltration cell in low- and high- risk group}

Previous studies showed that IRGs can influence the immune cell composition of tumor microenvironment. Therefore, to further understand the difference of immune infiltration cell between low- and high-risk groups, CIBERSORT package was used to calculate the 22 types of immune cells for each sample, and only samples with CIBERSORT $p<0.05$ were included in further study [33]. Then, Wilconson's rank sum test was used to compare 22 types of immune cells between the high-risk and low-risk groups.

\section{Results}

\section{Baseline}

According to the criteria, 255 soft tissue sarcoma patients were selected in our research. Then, 170 patients were incorporated into the training set and the remaining 85 patients were used to form the testing set. The baseline information of all patients shown in Table
1. The results indicated that the differences of clinical data between the training and testing sets were not statistically significant.

\section{Identification of prognostic IRGs in sarcoma patients}

To identify the prognostic value of IRGs in soft tissue sarcoma patients, univariate Cox proportional hazard model was performed in 170 patients. Totally, 105 IRGs were selected as OS-related IRGs (Supplementary 1). Moreover, GO and KEGG enrichment analyses were performed, and the results are shown in Fig. 1, which indicated that the major enriched $\mathrm{GO}$ terms of $\mathrm{BP}$ were defense response to other organism, positive regulation of cell adhesion, regulation of innate immune response, regulation of leukocyte activation, and positive regulation of leukocyte activation. In $\mathrm{CC}$, the major enriched GO terms were adherens junction, focal adhesion, cell -substrate adherens junction, cell-substrate junction and Schaffer collateral - CA1 synapse. For MF, we can find that the OS-related IRGs were mainly enriched in receptor ligand activity, receptor regulator activity, growth factor activity, cytokine activity, and steroid hormone receptor activity. For KEGG pathway analysis, it

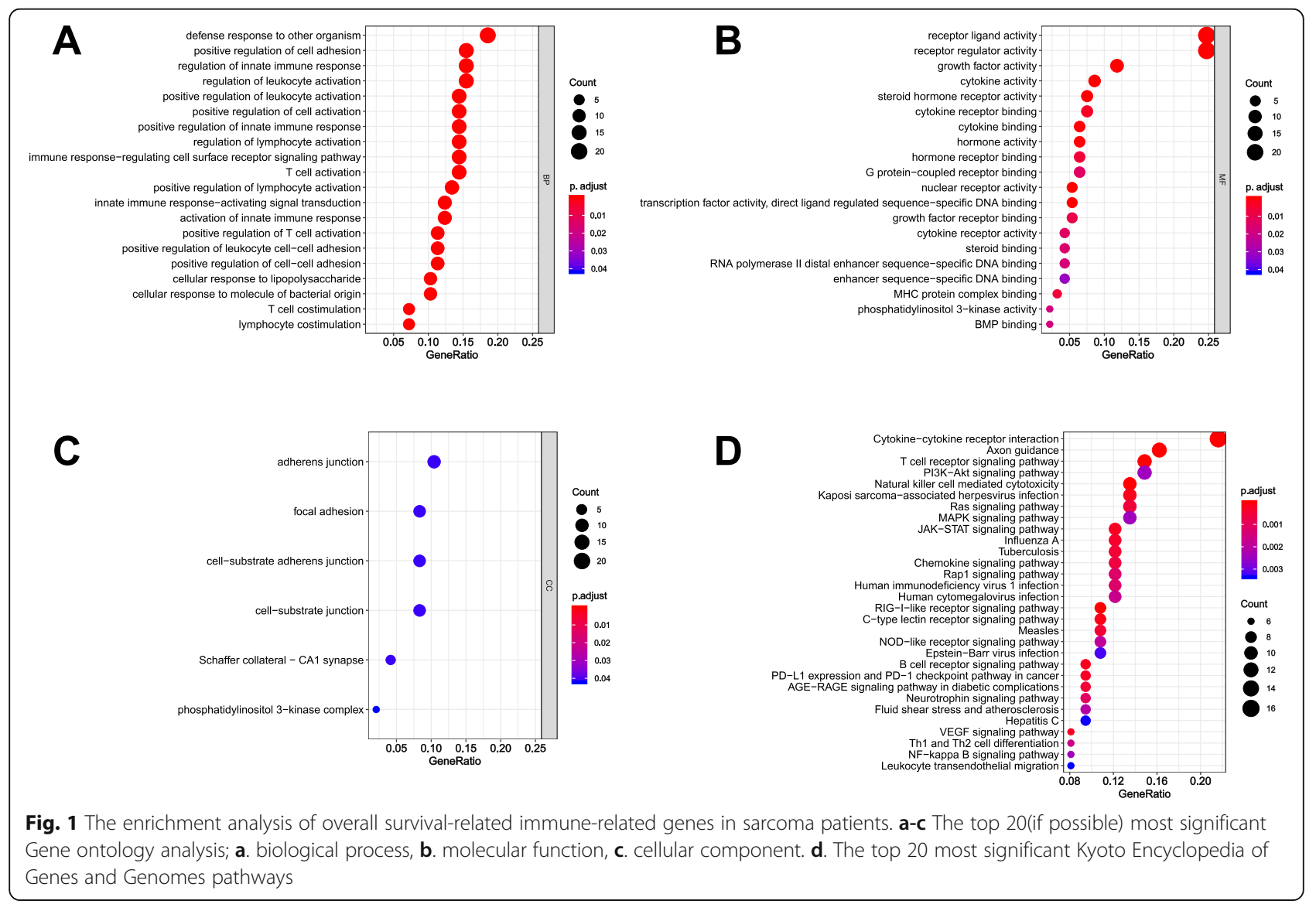


showed that many immune- or tumor-related pathways were identified, such as $\mathrm{T}$ cell receptor signaling pathway, Natural killer cell mediated cytotoxicity, Kaposi sarcoma-associated herpesvirus infection, PD - L1 expression and $\mathrm{PD}-1$ checkpoint pathway in cancer, Th1 and Th2 cell differentiation, and NF - kappa B signaling pathway.

\section{Construction of a TF regulatory network}

To elucidate regulatory mechanisms of OS-related IRGs, we used univariate Cox proportional hazard model to identify the OS-related TFs. Totally, 36 OS-related TFs were confirmed (Supplementary 2). Furthermore, we analyzed the correlation between the expression of OSrelated TFs and OS-related IRGs, the correlation results are shown in Supplementary 3. Interestingly, the results indicated that all TFs were positively correlated with IRGs. To better illustrate the regulatory relationship between TFs and IRGs, a TF-based regulatory network was generated (Fig. 2).

\section{Construction development and validation of the IRGs prognostic signature in training cohort}

Based on 105 OS-related IRGs, the LASSO regression was used to choose the best appropriate genes as the prognostic predictors of the model. Thirty-five genes were selected in the LASSO regression analysis (Fig. 3). Then, the multivariate Cox proportional hazard model was performed based on the significant genes in the LASSO analysis, and a prognostic signature was established based on the 19 prognostic IRGs (Supplementary 4 and Fig. 4). The time-dependent ROC of 1-, 2-, and 3-years were shown in Fig. 4c. The AUC values of 1-, 2-, and 3-years were 0.938, 0.937, and 0.935, respectively, which means that the prognostic signature can serve as a valid tool for prognostic prediction in sarcoma patients (Fig. 4c). In addition, the risk scores of each patient in the training set were calculated, the median of risk score was used as the cutoff to stratify patients into high-risk $(n=85)$ and low-risk $(n=85)$ groups. The survival curve was generated, and the logrank test indicated that the patients in the low-risk group had a favorable prognosis (Fig. 4d).

\section{External validation of IRGs signature}

To further verify the stability and reliability of the risk signature based on the IRGs, an independent set was used. As the formula of risk score in the training set, the risk scores of each patient sample in the testing set were calculated (Fig. 5). The time-dependent ROC curves were generated to test the discrimination of the signature (Fig. 5c). The results showed that the AUC values of $1-, 2-$, and 3 -year were $0.730,0.717$, and 0.647 , which also showed good accuracy of nomogram in predicting the OS of sarcoma patients. Furthermore, according to the median of the risk score in the testing set, 85 patients were stratified into the low-risk group $(n=43)$ and high-risk group $(n=42)$. The survival curve of two groups was generated, and the results indicated that
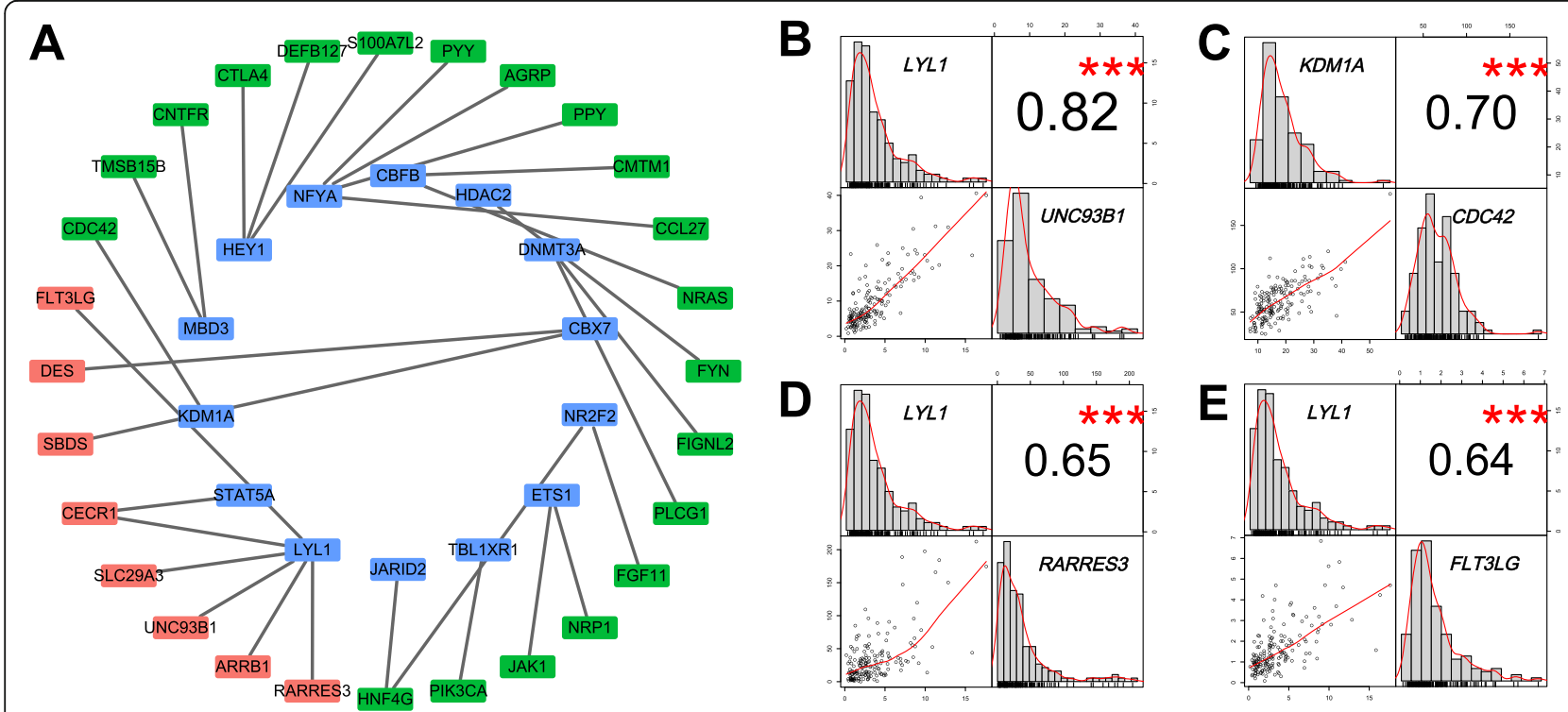

Fig. 2 Transcription factors-based regulatory network. a. Regulatory network of prognostic transcription factors and prognostic immune-related genes. The rectangle represent prognostic transcription factors, the green rectangle represent poor prognosis-related immune-related genes, and the red rectangle represent good prognosis-related immune-related genes. $\mathbf{b}$-e. Four examples to show the distribution of prognostic transcription factors and prognostic immune-related genes 


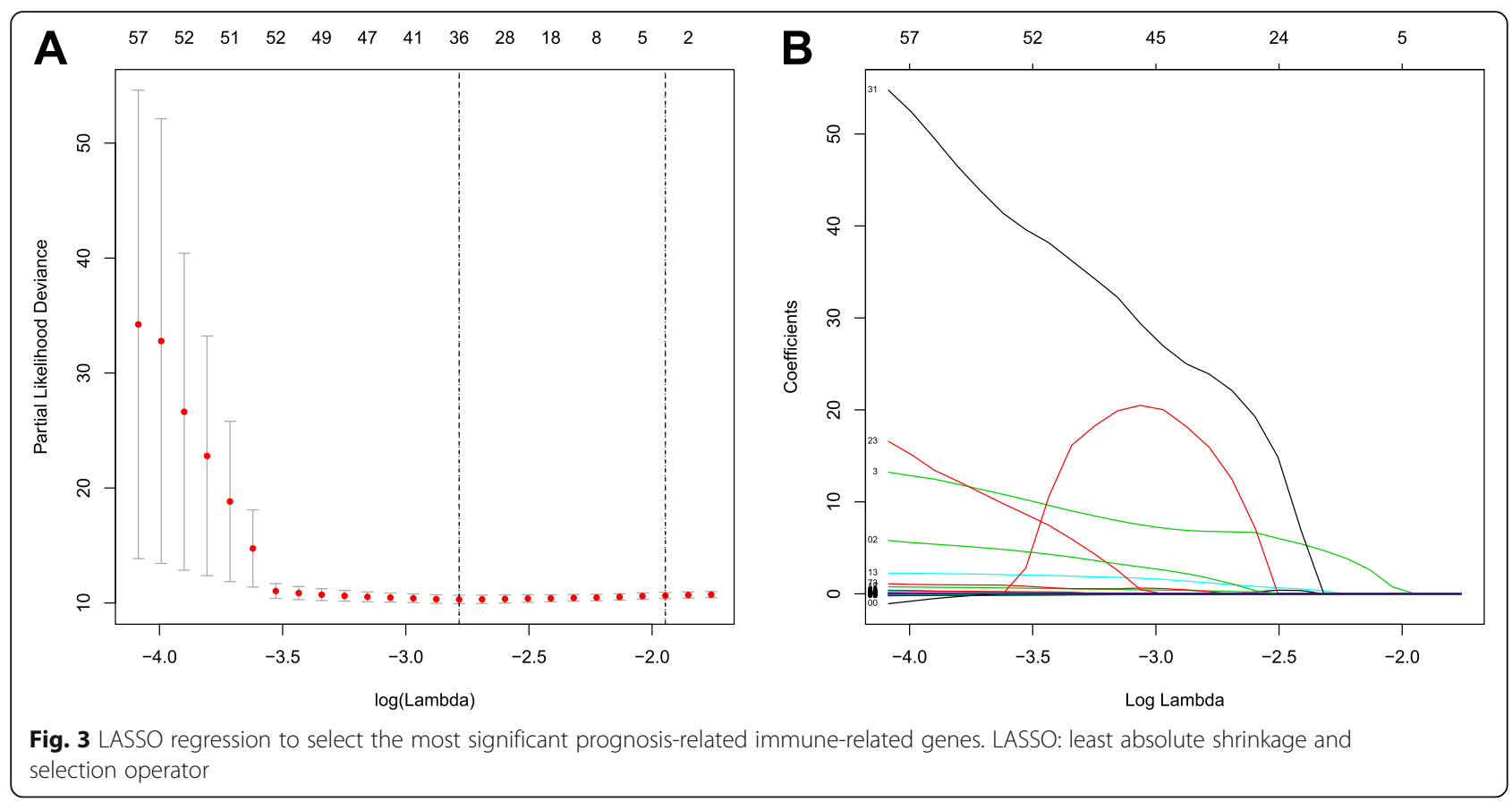

patients in the high-risk group have a worse prognosis (Fig. 5d).

\section{Development of a nomogram based on the IRG signature and clinical data}

To further construct a prognostic nomogram combining IRGs and clinical data, we performed univariate and multivariate Cox regression analysis to assess the independent prognostic variables for soft tissue sarcoma patients. In the univariate analysis, age, disease multifocal indicator, metastatic disease confirmed, surgical margin resection status and risk score were associated with the prognosis of sarcoma patients (Table 2). Then, the significant variables in the univariate Cox analysis were incorporated into the multivariate Cox analysis, and five independent prognostic variables were identified (Table 2). According to the results of the multivariate Cox analysis, we can find that risk score has the greatest impact for OS. In addition, higher age, multifocal sarcoma, tumor metastasis, and surgical resection status (R1-2) were also associated with worse prognosis in soft tissue sarcoma patients (Table 2). To better predict the prognosis of soft tissue sarcoma patients, we constructed a nomogram based on the independent factors determined in the multivariate regression (Fig. 6a). The C-index of our nomogram was 0.775 (95\%CI:0.751-0.799), which showed good accuracy in predicting the prognosis of soft tissue sarcoma patients. The favorable calibration plot of our nomogram indicated that the OS predicted by the nomogram is highly consistent with the actual observation (Fig. 6b-d). In addition, DCA was also performed, and the results indicated the nomogram can serve as an effective prognostic model for soft tissue sarcoma patients (Fig. 6e-g).

\section{Subgroup analyses of nomogram}

To further confirm that the nomogram can perform stably in different histological types of soft tissue sarcoma, patients were divided into different subgroups (Fig. 7). We can find the AUC values of the nomogram were higher than 0.750 (range:0.750-0.867) in all subgroups, which means that the nomogram can perform stably in different histological types of soft tissue sarcoma. In addition, in all subgroups, the Kaplan-Meier survival curves and the log-rank test indicated that the patients in the high-risk group have a poorer prognosis than patients in the low-risk group (all $p<0.05$ ) (Fig. 7).

\section{Comparison of the immune infiltration cell in low- and high-risk group}

After CIBERSORT package was performed, 169 patients with complete data of immune infiltration cells were included in this part of the study (Fig. 8a). Among the 169 patients, 87 were in the low-risk group and 82 were in the high-risk group. The results showed that six immune cells were significantly different between the two groups (Fig. 8b). The infiltration level of plasma cells and macrophages M0 were significantly higher in the high-risk group, while the infiltration level of NK cells resting, NK cells activated, monocytes, and macrophages M1 were significantly higher in the low-risk group (Fig. 8b). 


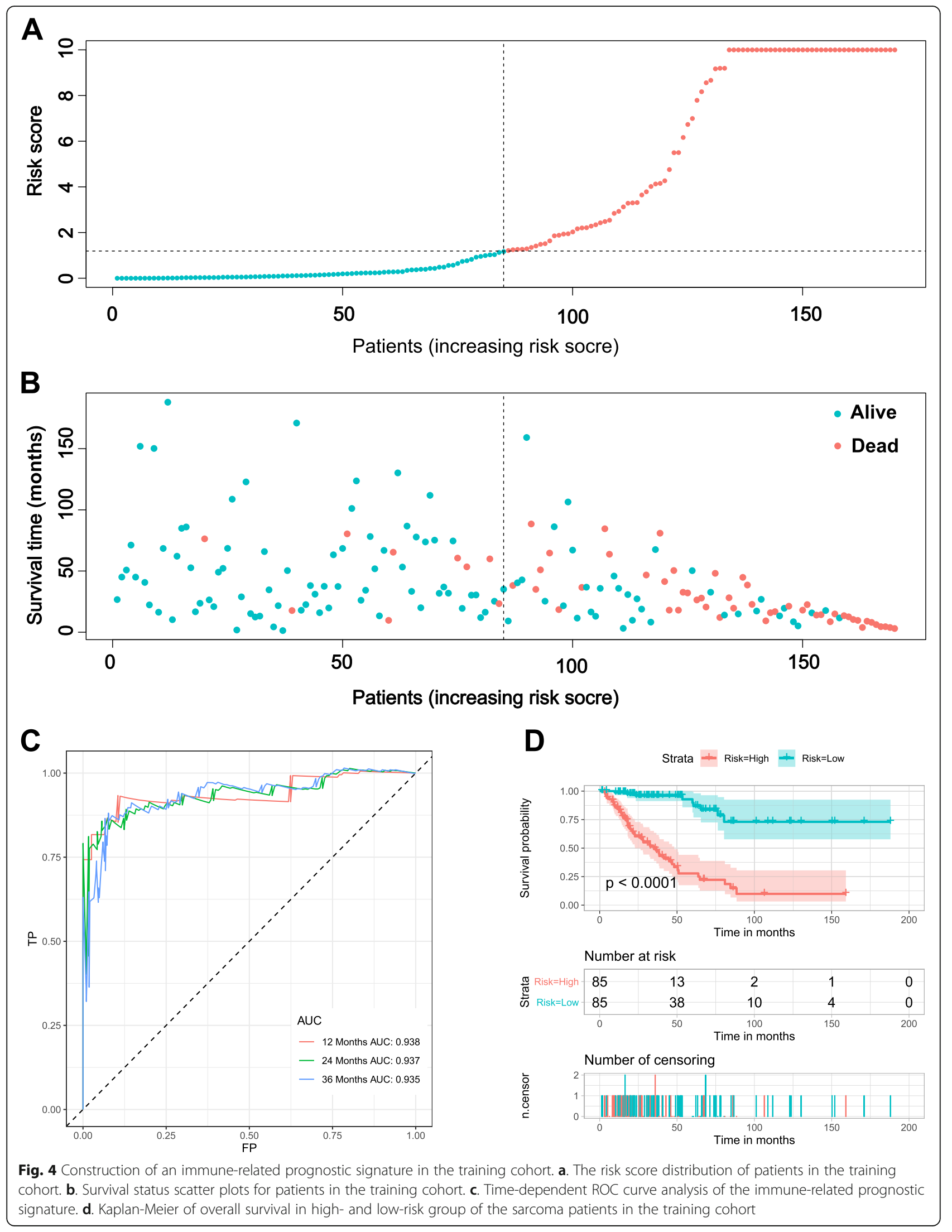




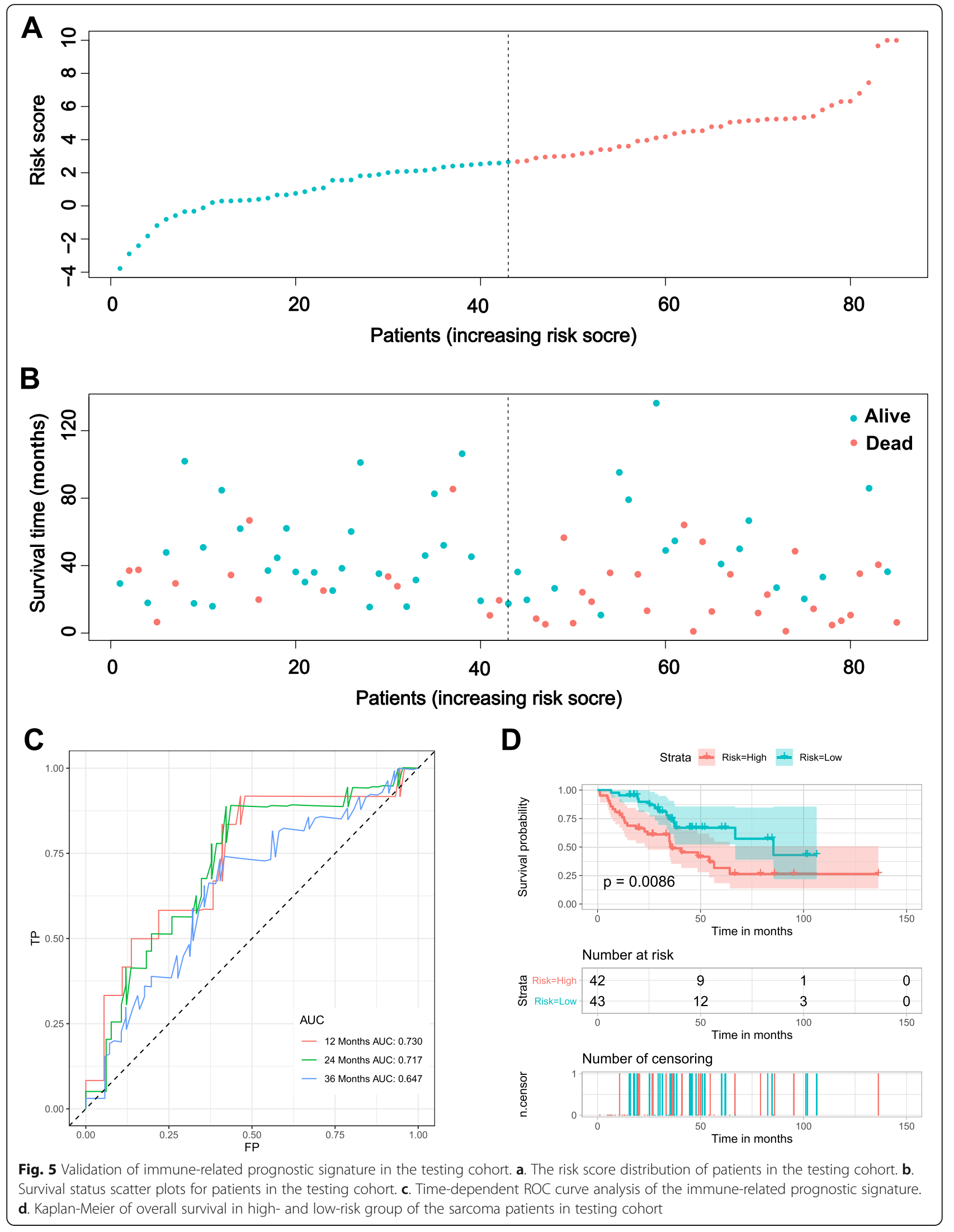


Table 2 Univariate and multivariate Cox analysis in sarcoma patients

\begin{tabular}{|c|c|c|c|c|c|c|c|c|}
\hline \multirow{3}{*}{ Age } & \multicolumn{4}{|c|}{ Univariate analysis } & \multicolumn{4}{|c|}{ Multivariate analysis } \\
\hline & \multirow{2}{*}{$\begin{array}{l}\text { HR } \\
1.020\end{array}$} & \multicolumn{2}{|c|}{$95 \% \mathrm{Cl}$} & \multirow{2}{*}{$\begin{array}{l}\boldsymbol{P} \\
0.012\end{array}$} & \multirow{2}{*}{$\begin{array}{l}\mathbf{H R} \\
1.025\end{array}$} & \multicolumn{2}{|c|}{$95 \% \mathrm{Cl}$} & \multirow{2}{*}{$\frac{P}{0.004}$} \\
\hline & & 1.004 & 1.036 & & & 1.008 & 1.043 & \\
\hline \multicolumn{9}{|l|}{ Sex } \\
\hline \multicolumn{9}{|l|}{ Female } \\
\hline Male & 0.849 & 0.565 & 1.274 & 0.429 & & & & \\
\hline \multicolumn{9}{|l|}{ Race } \\
\hline Asian & & & & 0.412 & & & & \\
\hline African American & 1.108 & 0.135 & 9.098 & 0.924 & & & & \\
\hline WHITE & 0.810 & 0.111 & 5.907 & 0.836 & & & & \\
\hline Unknown & 2.004 & 0.207 & 19.378 & 0.548 & & & & \\
\hline \multicolumn{9}{|l|}{ Histological type } \\
\hline DLP & & & & 0.903 & & & & \\
\hline LMS & 0.855 & 0.515 & 1.419 & 0.543 & & & & \\
\hline MYX & 0.730 & 0.339 & 1.571 & 0.421 & & & & \\
\hline UPS & 0.960 & 0.509 & 1.810 & 0.899 & & & & \\
\hline Other & 0.746 & 0.321 & 1.734 & 0.496 & & & & \\
\hline \multicolumn{9}{|l|}{ Multifocal indicator } \\
\hline No & & & & 0.002 & & & & 0.008 \\
\hline Yes & 2.328 & 1.443 & 3.754 & 0.001 & 2.228 & 1.324 & 3.748 & 0.003 \\
\hline Unknown & 1.220 & 0.606 & 2.459 & 0.577 & 1.135 & 0.486 & 2.651 & 0.770 \\
\hline \multicolumn{9}{|l|}{ Metastasis } \\
\hline No & & & & $<0.001$ & & & & $<0.001$ \\
\hline Yes & 2.962 & 1.797 & 4.880 & $<0.001$ & 2.926 & 1.721 & 4.972 & $<0.001$ \\
\hline Unknown & 1.795 & 1.078 & 2.987 & 0.024 & 1.770 & 1.005 & 3.115 & 0.048 \\
\hline \multicolumn{9}{|l|}{ Radiotherapy } \\
\hline No & & & & 0.963 & & & & \\
\hline Yes & 1.012 & 0.633 & 1.620 & 0.959 & & & & \\
\hline Unknown & 1.075 & 0.638 & 1.812 & 0.786 & & & & \\
\hline \multicolumn{9}{|l|}{ Surgical margin resection status } \\
\hline RO & & & & $<0.001$ & & & & 0.005 \\
\hline $\mathrm{R} 1-2$ & 2.418 & 1.572 & 3.719 & $<0.001$ & 1.974 & 1.257 & 3.099 & 0.003 \\
\hline Unknown & 2.194 & 1.156 & 4.165 & 0.016 & 2.099 & 0.960 & 4.589 & 0.063 \\
\hline \multicolumn{9}{|l|}{ Tumor site } \\
\hline Extremity & & & & 0.745 & & & & \\
\hline Other & 1.182 & 0.699 & 1.997 & 0.533 & & & & \\
\hline Retroperitoneum/Upper abdominal & 1.193 & 0.735 & 1.934 & 0.475 & & & & \\
\hline Risk & 5.192 & 3.215 & 8.382 & $<0.001$ & 5.362 & 3.241 & 8.868 & $<0.001$ \\
\hline
\end{tabular}

LMS Leiomyosarcoma, DLP Dedifferentiated liposarcoma, UPS Undifferentiated pleomorphic sarcoma, MYX Myxofibrosarcoma

Additionally, as macrophage plays a key role in influencing the anti-tumor immune responses, we further analyzed the infiltration of macrophages M1 and M2 in the low-risk groups. The results showed that the infiltration level of macrophages M2 were significantly higher than the infiltration level of macrophages M1 (Fig. 8c).

\section{Discussion}

Due to the heterogeneity and high metastatic rate of sarcoma, treatment methods were very limited and the outcome was unfavorable. Therefore, it is necessary for us to identify the effective biomarkers for the prognosis of sarcoma patients. In the present study, 105 IRGs were identified as prognostic IRGs and the regulatory network 


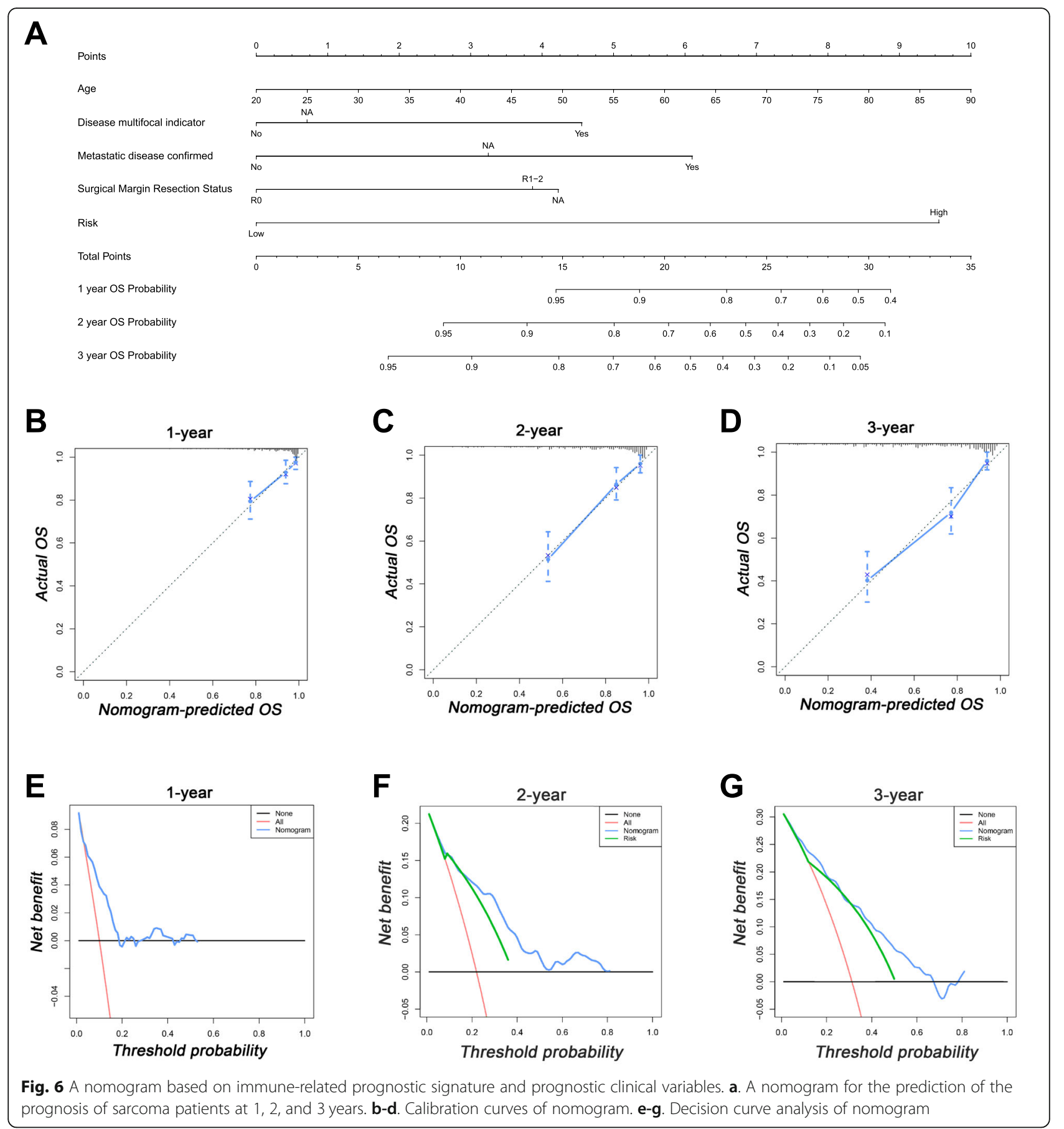

between prognostic IRGs and TFs was established. More importantly, we constructed a prognostic signature based on 19 IRGs and validated its effectiveness, which also was proved to be associated with immune infiltration cells. Furthermore, a nomogram incorporating IRGbased signature and clinical data was established, which showed excellent performance to predict the outcome of sarcoma patients.
With the wide exploration of immune mechanisms in the pathogenesis and progression of the tumor, many IRGs were identified as prognostic biomarkers in tumor patients [17-20, 23-26, 34]. More importantly, based on the researches of tumor immunology, many immunotherapies have been developed and become new methods for treating sarcoma patients, and results indicated that the outcome was satisfactory [35-37]. 


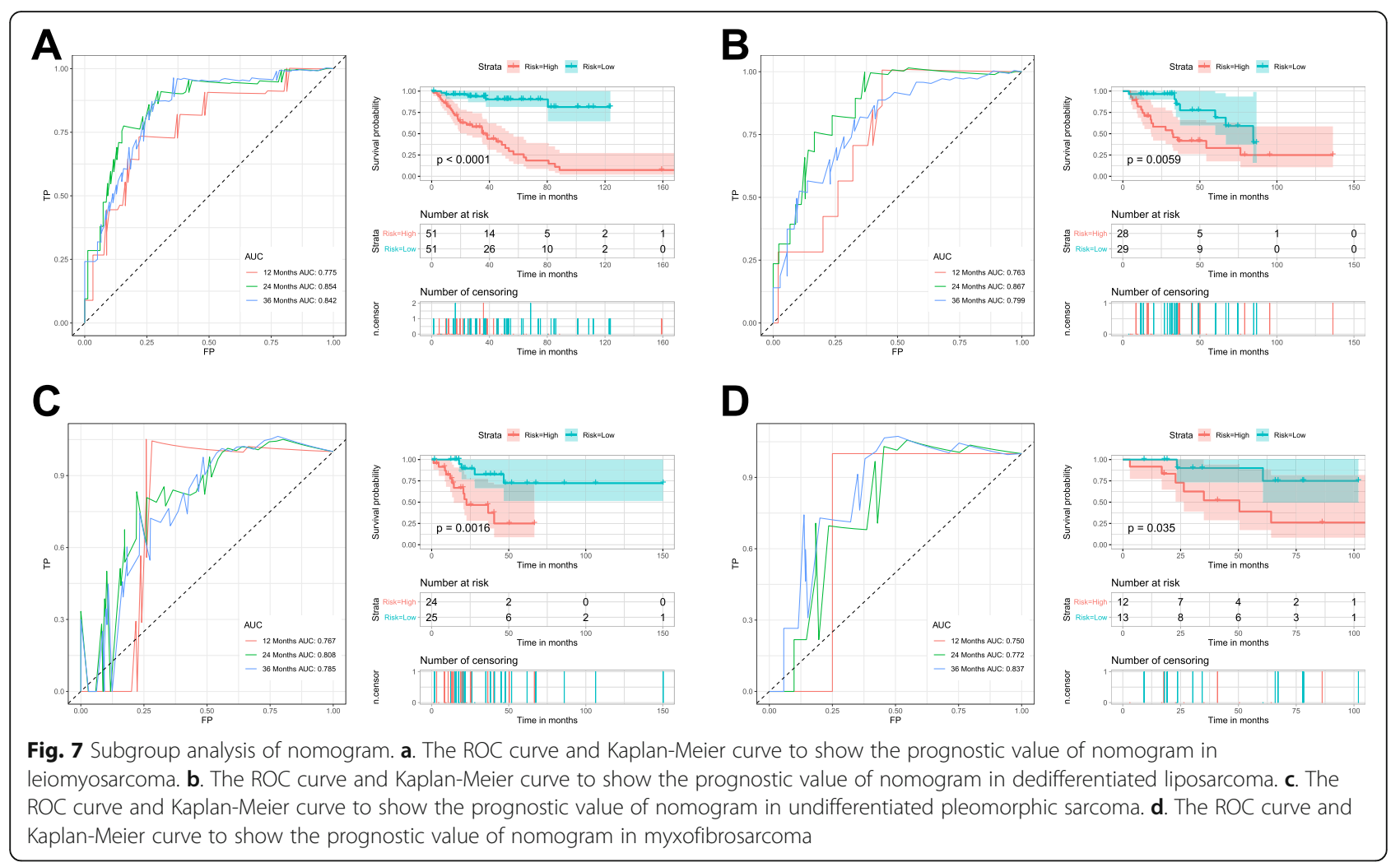

However, as far as we know, it is the first research to establish a signature based on IRGs for sarcoma patients, which may open up a novel perspective for constructing an effective prognostic model and improving sarcoma patient management of immunotherapy.

In our research, a prognostic signature incorporating 19 IRGs was established. The AUC of the model was higher than 0.9 in the training set, which showed great accuracy in predicting the prognosis of sarcoma patients. Moreover, the accuracy of our model was also successfully verified by an independent set. Our results indicated that the model can be used to identify sarcoma patients at high risk and enabling early interventions to improve the prognosis. Among 19 IRGs enrolled in risk signature, VEGFA, CYR61, and RHOA have confirmed to be related to the pathogenesis or prognosis of sarcoma [38-41]. It was reported that VEGFA is a potential neovascular regulatory factor that promotes tumor growth and metastasis through its receptor. Differential expression of VEGFA subtypes regulates sarcoma metastasis and response to anti-VEGFA [38]. Besides, the VEGF family can not only promote tumor-associated immunodeficiency by interfering with the growth of early hematopoietic progenitor $\mathrm{T}$ cells, but promote Treg cell proliferation [42, 43]. CYR61 is a member of the CCN family. The expression level of CYR61 is associated with the aggressiveness of osteosarcoma in different pre-clinical models and patient tumor samples, and
CYR61 triggers osteosarcoma metastasis and spread through an IGF1R $\beta$-dependent EMT-like process [44]. The metastasis of invasive amoebic sarcoma cells depends on the Rho / Rock / MLC signal, and RHOA overexpression is related to tumor cell invasion and migration [41]. The relationship between the remaining 16 genes and sarcoma has few been reported, however, most of them have been confirmed to be associated with other tumors, which suggested that these genes also play essential roles in sarcoma. For example, PSMD10 is a crucial oncoprotein that is up-regulated in a variety of cancers and has potential in the initiation and progression of tumors [45]. Studies have indicated important roles played by PSMD10 in the pathogenesis of liver cancers and colorectal cancers, and PSMD 10 involved in regulating the proliferation and metastasis of thyroid cancers [46].

Combining the results of the above KEGG pathway and immune genes revealed the existence of potential immune mechanisms in sarcoma. Previous studies have suggested that high levels of macrophage infiltration predict a poor prognosis for Ewing's sarcoma, which is consistent with the result that macrophage M0 significantly increased in high-risk groups in our study [47]. Additionally, different subtypes of macrophage showed different infiltration model and may serve as different role in cancer progression and anti-tumor immune responses. Macrophage M1 not only associated with the 


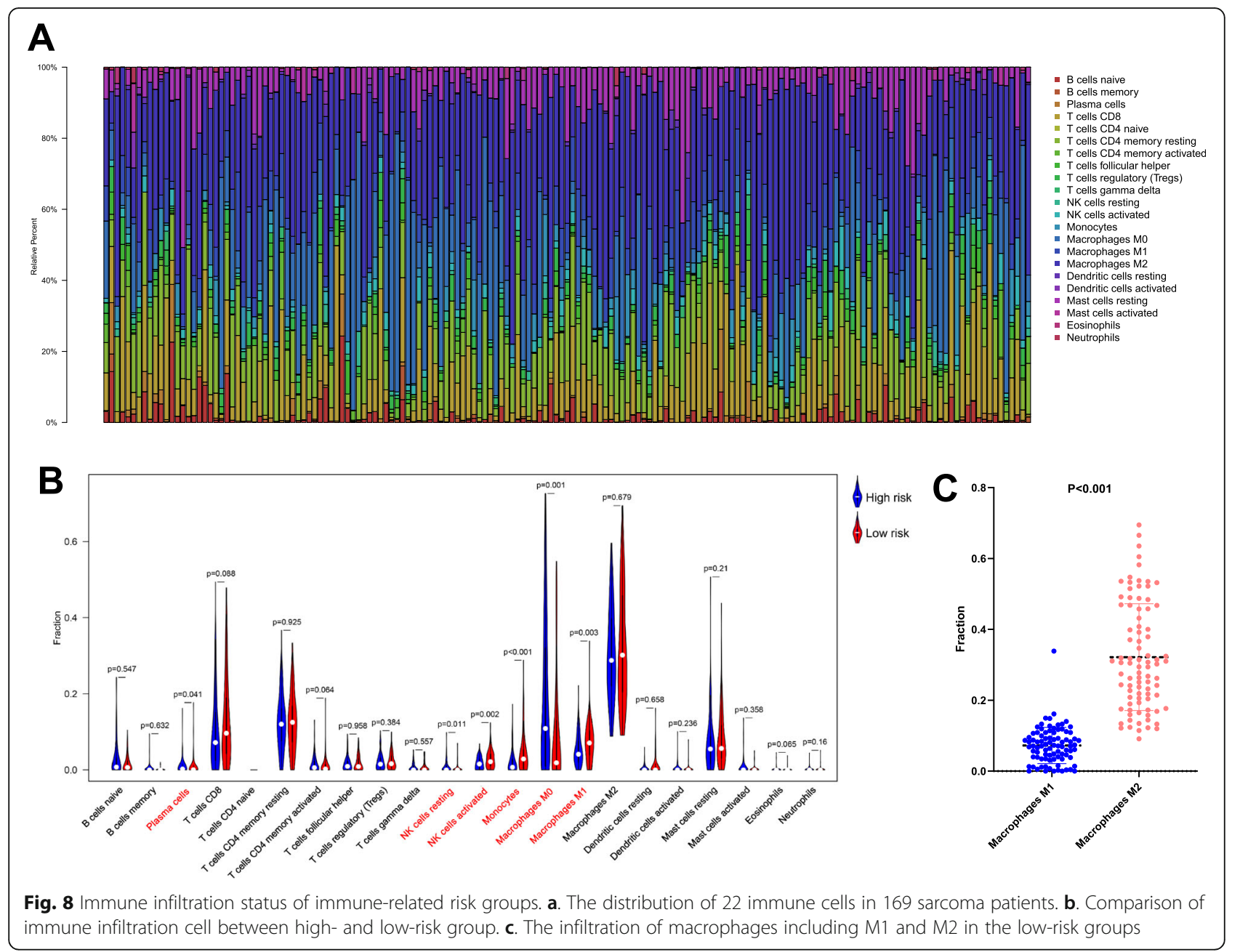

OS of sarcoma, but also significantly associated with recurrence of sarcoma [48]. macrophages M2 were found to be the most abundant immune cell type and were associated with improved survival in osteosarcoma [49]. Interestingly, Dhupkar et.al indicated that osteosarcoma lung metastases regression by anti-PD1 can be attributed to activated tumor macrophages $\mathrm{M} 1$ and reduced macrophages M2 [50]. Although there are few studies focused on macrophages M0, its relationship with other immune cells, such as CD8 T cells, has also been preliminarily reported [51]. Natural killer (NK) cells are important immune cells in the innate immune mechanisms. The activation of NK cells can promote the immunotherapy of Ewing's sarcoma [52]. In this study, we found that activated NK cells were higher in the low-risk group and enriched in the KEGG pathway, which can provide a theoretical basis for immunotherapy in sarcoma patients.

In addition, in order to make the clinical application more convenient and accurate, a nomogram was established by combining immune signature and clinical data.
The results of the AUC, calibration curve, DCA and subgroup analysis indicated that the nomogram can serve as an effective tool for predicting the prognosis in sarcoma patients. Although a lot of predictive models for sarcoma patients were constructed based on the clinicopathologic data, lncRNA, plasmacytoma variant translocation 1 and other predictors $[13,53,54]$. However, it should point out that the discriminative ability of the previous models was low with AUC or C-statistic less than 0.750 [13, 5355], which means that they are unsatisfactory. Therefore, we think that the IRG-based nomogram can improve the risk stratification for sarcoma patients.

There are some limitations to our research. Firstly, this is a retrospective study, which may lead to bias and a novel sight and large cohort to verify is needed. Second, although we find the prognostic ability of the signature and develop a nomogram with great predictive ability, more clinical features are needed to improve the nomograms, such as AJCC TNM stage. Thirdly, this study was a bioinformatic study based on a public database, and the experiment should be 
performed in the future to explore the mechanism of the effects of IRGs in the prognosis of sarcoma. Finally, the training and testing sets were essentially from one cohort and the prognostic signature has no real external validation. Therefore, the validation of this prognostic model was relative weak and further validation in independent cohort is needed.

\section{Conclusion}

In summary, we identified and validated an IRG-based signature, which can be used as an independent prognostic signature in evaluating the prognosis of sarcoma patients. However, further experimental exploration is needed to study the potential mechanism of IRGs in sarcoma.

\section{Supplementary Information}

The online version contains supplementary material available at https://doi. org/10.1186/s12885-021-07852-2.

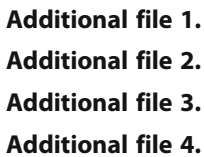

\section{Abbreviations}

IRGs: Immune-related genes; TCGA: The Cancer Genome Atlas; ROC: Receiver operating characteristic; AUC: Area under the curve; RNA-seq: RNAsequencing; OS: Overall survival; GO: Gene Ontology; MF: Molecular function; BP: Biological process; CC: Cellular component; KEGG: Kyoto Encyclopedia of Genes and Genomes; K-M: Kaplan-Meier; DCA: Decision curve analysis; NK: Natural killer; BCR: B cell antigen receptor; PD1: Programmed cell death 1; PDL-1: Programmed Death Ligand-1

\section{Acknowledgements}

None.

\section{Authors' contributions}

R S, JF M and TB Y conceived and designed the study. R S and B L performed literature search. R S and XS L generated the figures and tables. $\mathrm{KS} X$ and $B L$ analyzed the data. R S wrote the manuscript and JF M critically reviewed the manuscript. JF $M$ and TB $Y$ supervised the research. All authors have read and approved the manuscript.

\section{Funding}

We received no external funding for this study.

\section{Availability of data and materials}

The data of this study are from The Cancer Genome Atlas (https://portal.gdc. cancer.gov/) and ImmPort database (https://www.immport.org/).

\section{Ethics approval and consent to participate}

The research didn't involve animal experiments and human specimens, no ethics related issues.

\section{Consent for publication}

Not applicable.

\section{Competing interests}

The authors declare that they have no competing interests.

\section{Author details}

'Department of Spinal Surgery, the Affiliated Hospital of Qingdao University, Qingdao 266000, China. ${ }^{2}$ Department of Sport Medicine, The Affiliated Hospital of Qingdao University, Qingdao 266000, China.

Received: 11 August 2020 Accepted: 27 January 2021

Published online: 08 February 2021

\section{References}

1. Toro JR, Travis LB, Wu HJ, Zhu K, Fletcher CDM, Devesa SS. Incidence patterns of soft tissue sarcomas, regardless of primary site, in the surveillance, epidemiology and end results program, 1978-2001: an analysis of 26,758 cases. Int J Cancer. 2006:119(12):2922-30.

2. CDM F. The evolving classification of soft tissue tumours - an update based on the new 2013 WHO classification. Histopathology. 2014;64:1.

3. Cormier JN, Huang X, Xing Y, Thall PF, Wang X, Benjamin RS, et al. Cohort analysis of patients with localized, high-risk, extremity soft tissue sarcoma treated at two cancer centers: chemotherapy-associated outcomes. J Clin Oncol. 2004;22(22):4567-74.

4. Lewis DR, Seibel NL, Smith AW, Stedman MR. Adolescent and young adult cancer survival. J Natl Cancer Inst Monographs. 2014;2014(49):228-35.

5. Siegel RL, Miller KD, Jemal A. Cancer statistics, 2018. CA Cancer J Clin. 2018; 68:1.

6. Kim HS, Nam CM, Jang S-Y, Choi SK, Han M, Kim S, et al. Characteristics and treatment patterns of patients with advanced soft tissue sarcoma in Korea. Cancer Res Treat. 2019:51(4):1380-91.

7. Schöffski P, Cornillie J, Wozniak A, Li H, Hompes D. Soft tissue sarcoma: an update on systemic treatment options for patients with advanced disease. Oncol Res Treat. 2014;37(6):355-62.

8. Kollár A, Rothermundt C, Klenke F, Bode B, Baumhoer D, Arndt V, et al. Incidence, mortality, and survival trends of soft tissue and bone sarcoma in Switzerland between 1996 and 2015. Cancer Epidemiol. 2019;63:101596.

9. Bessen T, Caughey GE, Shakib S, Potter JA, Reid J, Farshid G, et al. A population-based study of soft tissue sarcoma incidence and survival in Australia: an analysis of 26,970 cases. Cancer Epidemiol. 2019:63:101590.

10. Italiano A, Mathoulin-Pelissier S, Cesne AL, Terrier P, Bonvalot S, Collin F, et al. Trends in survival for patients with metastatic soft-tissue sarcoma. Cancer. 2011;117(5):1049-54

11. Min L, Garbutt C, Tu C, Hornicek F, Duan Z. Potentials of Long noncoding RNAs (LnCRNAs) in sarcoma: from biomarkers to therapeutic targets. Int J Mol Sci. 2017;18:4

12. Kohama I, Kosaka N, Chikuda H, Ochiya T. An insight into the roles of MicroRNAs and Exosomes in sarcoma. Cancers. 2019;11:3.

13. Liu J, Li R, Liao X, Hu B, Yu J. Comprehensive investigation of the clinical significance and molecular mechanisms of plasmacytoma variant translocation 1 in sarcoma using genome-wide RNA sequencing data. J Cancer. 2019;10(20):4961

14. Hu C, Wang Y, Liu C, Shen R, Chen B, Sun K, et al. Systematic profiling of alternative splicing for sarcoma patients reveals novel prognostic biomarkers associated with tumor microenvironment and immune cells. Med Sci Monit. 2020;26:e924126.

15. Gentles AJ, Newman AM, Liu CL, Bratman SV, Feng W, Kim D, et al. The prognostic landscape of genes and infiltrating immune cells across human cancers. Nat Med. 2015;21(8):938-45.

16. Zuo W, Zhao L. Recent advances and application of PD-1 blockade in sarcoma. Onco Targets Ther. 2019;12:6887-96

17. Oiu H, Hu X, He C, Yu B, Li Y, Li J. Identification and validation of an individualized prognostic signature of bladder cancer based on seven immune related genes. Front Genet. 2020:11:12

18. Shen C, Liu J, Wang J, Zhong X, Dong D, Yang X, et al. Development and validation of a prognostic immune-associated gene signature in clear cell renal cell carcinoma. Int Immunopharmacol. 2020;81:106274.

19. He Y, Dang Q, Li J, Zhang Q, Yu X, Xue M, et al. Prediction of hepatocellular carcinoma prognosis based on expression of an immune-related gene set Aging (Albany NY). 2020;12(1):965.

20. Wang Z, Zhu J, Liu Y, Liu C, Wang W, Chen F, et al. Development and validation of a novel immune-related prognostic model in hepatocellular carcinoma. J Transl Med. 2020;18(1):67.

21. Luo J, Liu P, Wang L, Huang Y, Wang Y, Geng W, et al. Establishment of an immune-related gene pair model to predict colon adenocarcinoma prognosis. BMC Cancer. 2020;20(1):1071. 
22. Liu C, Chen B, Huang Z, Hu C, Jiang L, Zhao C. Comprehensive analysis of a 14 immune-related gene pair signature to predict the prognosis and immune features of gastric cancer. Int Immunopharmacol. 2020;89:107074.

23. Li B, Cui Y, Diehn M, Li R. Development and validation of an individualized immune prognostic signature in early-stage nonsquamous non-small cell lung cancer. JAMA Oncol. 2017;3(11):1529-37.

24. Song Q, Shang J, Yang Z, Zhang L, Zhang C, Chen J, et al. Identification of an immune signature predicting prognosis risk of patients in lung adenocarcinoma. J Transl Med. 2019;17(1):70

25. Shi $X$, Li R, Dong X, Chen AM, Liu X, Lu D, et al. IRGS: an immune-related gene classifier for lung adenocarcinoma prognosis. J Transl Med. 2020;18(1): $1-11$.

26. Zhang M, Zhu K, Pu H, Wang Z, Zhao H, Zhang J, et al. An immune-related signature predicts survival in patients with lung adenocarcinoma. Front Oncol. 2019;9:13-4.

27. Yu G, Wang L-G, Han Y, He Q-Y. clusterProfiler: an R package for comparing biological themes among gene clusters. OMICS. 2012;16(5):284-7.

28. Shannon P, Markiel A, Ozier O, Baliga NS, Wang JT, Ramage D, et al. Cytoscape: a software environment for integrated models of biomolecular interaction networks. Genome Res. 2003;13(11):2498-504.

29. Engebretsen S, Bohlin J. Statistical predictions with glmnet. Clin Epigenetics. 2019;11(1):123.

30. Ranstam J, Cook J. Kaplan-Meier curve. Br J Surg. 2017:104:4.

31. Harrell FE Jr. Rms: regression modeling strategies. R package version. 2016;5:2.

32. Vickers AJ, Elkin EB. Decision curve analysis: a novel method for evaluating prediction models. Med Decis Mak. 2006;26(6):565-74.

33. Newman AM, Liu CL, Green MR, Gentles AJ, Feng W, Xu Y, et al. Robust enumeration of cell subsets from tissue expression profiles. Nat Methods. 2015;12(5):453-7.

34. Shen S, Wang G, Zhang R, Zhao Y, Yu H, Wei Y, et al. Development and validation of an immune gene-set based prognostic signature in ovarian cancer. EBioMedicine. 2019;40:318-26.

35. Gouw LG, Jones KB, Sharma S, Randall RL. Sarcoma immunotherapy. Cancers. 2011;3(4):4139-50.

36. Mackall CL, Rhee EH, Read EJ, Khuu HM, Leitman SF, Bernstein D, et al. A pilot study of consolidative immunotherapy in patients with high-risk pediatric sarcomas. Clin Cancer Res. 2008;14(15):4850-8.

37. Petitprez F, de Reyniès A, Keung EZ, Chen TW-W, Sun C-M, Calderaro J, et al. $B$ cells are associated with survival and immunotherapy response in sarcoma. Nature. 2020;577(7791):556-60.

38. English WR, Lunt SJ, Fisher M, Lefley DV, Dhingra M, Lee $Y$-C, et al. Differential expression of VEGFA isoforms regulates metastasis and response to anti-VEGFA therapy in sarcoma. Cancer Res. 2017;77(10):2633-46.

39. Croci S, Landuzzi L, Astolfi A, Nicoletti G, Rosolen A, Sartori F, et al. Inhibition of connective tissue growth factor (CTGF/CCN2) expression decreases the survival and myogenic differentiation of human rhabdomyosarcoma cells. Cancer Res. 2004;64(5):1730-6.

40. Manara MC, Perbal B, Benini S, Strammiello R, Cerisano V, Perdichizzi S, et al. The expression of ccn3(nov) gene in musculoskeletal tumors. Am J Pathol. 2002;160(3):849-59.

41. Kosla J, Paňková D, Plachý J, Tolde O, Bicanová K, Dvořák M, et al. Metastasis of aggressive amoeboid sarcoma cells is dependent on rho/ROCK/MLC signaling. Cell Commun Signal. 2013;11:51.

42. Ohm JE, Gabrilovich DI, Sempowski GD, Kisseleva E, Parman KS, Nadaf S, et al. VEGF inhibits T-cell development and may contribute to tumorinduced immune suppression. Blood. 2003;101(12):4878-86.

43. Tada Y, Togashi Y, Kotani D, Kuwata T, Sato E, Kawazoe A, et al. Targeting VEGFR2 with Ramucirumab strongly impacts effector/ activated regulatory T cells and CD8 T cells in the tumor microenvironment. J Immunother Cancer. 2018;6(1):106.

44. Habel N, Stefanovska B, Carène D, Patiño-Garcia A, Lecanda F, Fromigué O. CYR61 triggers osteosarcoma metastatic spreading via an IGF1Rßdependent EMT-like process. BMC Cancer. 2019;19(1):62.

45. Fujita J, Sakurai T. The Oncoprotein Gankyrin/PSMD10 as a target of cancer therapy. Adv Exp Med Biol. 2019;1164:63-71

46. Liu F, Lou K, Zhao X, Zhang J, Chen W, Qian Y, et al. miR-214 regulates papillary thyroid carcinoma cell proliferation and metastasis by targeting PSMD10. Int J Mol Med. 2018:42(6):3027-36.

47. Fujiwara T, Fukushi J-i, Yamamoto S, Matsumoto Y, Setsu N, Oda Y, et al. Macrophage infiltration predicts a poor prognosis for human Ewing sarcoma. Am J Pathol. 2011;179(3):1157-70.
48. Huang R, Meng T, Chen R, Yan P, Zhang J, Hu P, et al. The construction and analysis of tumor-infiltrating immune cell and ceRNA networks in recurrent soft tissue sarcoma. Aging. 2019;11(22):10116-43.

49. Deng $\mathrm{C}, \mathrm{Xu} Y, \mathrm{Fu} J$, Zhu X, Chen $\mathrm{H}, \mathrm{Xu} \mathrm{H}$, et al. Reprograming the tumor immunologic microenvironment using neoadjuvant chemotherapy in osteosarcoma. Cancer Sci. 2020;111(6):1899-909.

50. Dhupkar P, Gordon N, Stewart J, Kleinerman ES. Anti-PD-1 therapy redirects macrophages from an M2 to an M1 phenotype inducing regression of OS lung metastases. Cancer Med. 2018;7(6):2654-64.

51. Zhu N, Hou J. Assessing immune infiltration and the tumor microenvironment for the diagnosis and prognosis of sarcoma. Cancer Cell Int. 2020;20(1):577

52. Verhoeven DHJ, de Hooge ASK, Mooiman ECK, Santos SJ, ten Dam MM, Gelderblom $H$, et al. NK cells recognize and lyse Ewing sarcoma cells through NKG2D and DNAM-1 receptor dependent pathways. Mol Immunol. 2008:45(15):3917-25.

53. He R-q, Q-j W, R-x T, W-j C, Yang X, Peng Z-G, et al. Prediction of clinical outcome and survival in soft-tissue sarcoma using a ten-IncRNA signature. Oncotarget. 2017:8(46):80336

54. Callegaro D, Miceli R, Bonvalot S, Ferguson P, Strauss DC, Levy A, et al. Development and external validation of two nomograms to predict overall survival and occurrence of distant metastases in adults after surgical resection of localised soft-tissue sarcomas of the extremities: a retrospective analysis. Lancet Oncol. 2016:17(5):671-80.

55. Tape TG. Interpretation of diagnostic tests. Ann Intern Med. 2001;135(1):72.

\section{Publisher's Note}

Springer Nature remains neutral with regard to jurisdictional claims in published maps and institutional affiliations.
Ready to submit your research? Choose BMC and benefit from:

- fast, convenient online submission

- thorough peer review by experienced researchers in your field

- rapid publication on acceptance

- support for research data, including large and complex data types

- gold Open Access which fosters wider collaboration and increased citations

- maximum visibility for your research: over $100 \mathrm{M}$ website views per year

At $\mathrm{BMC}$, research is always in progress.

Learn more biomedcentral.com/submissions 